\title{
Inconsistent Adoption of World Health Organization V (2010) Semen Analysis Reference Ranges in the United States Six Years After Publication
}

Kevin C. Lewis BA ${ }^{a}$, Isaac Lam BA ${ }^{a}$, Jacob Nieb BS/BA ${ }^{a}$, Grace Lam BS ${ }^{b}$, Anuj S. Desai MD ${ }^{a}$, Daniel J. Mazur MDa ${ }^{a}$, Barbara Kahn $\mathrm{MD}^{\mathrm{a}}$, Mary Kate Keeter $\mathrm{MPH}^{\mathrm{a}}$, Alex Tatem $\mathrm{MD}^{\mathrm{c}}$, Marah Hehemann MD $^{d}$, Brendan T. Frainey MD ${ }^{a}$, Nelson Bennett, Jr. MD ${ }^{a}$, Robert E. Brannigan MD $^{a}$

${ }^{a}$ Northwestern University, Feinberg School of Medicine, Department of Urology, Chicago, IL, USA; 'Massachusetts Institute of Technology, Department of Electrical Engineering and Computer Science, Cambridge, MA, USA; 'Indiana University-Purdue University Indianapolis,

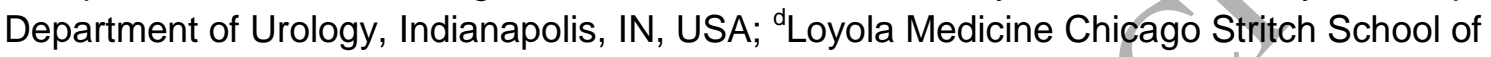
Medicine, Chicago, IL, USA

Keywords: Infertility, laboratory testing, semen analysis, world health organization

Address for Correspondence: $\quad$ Robert E. Brannigan MD

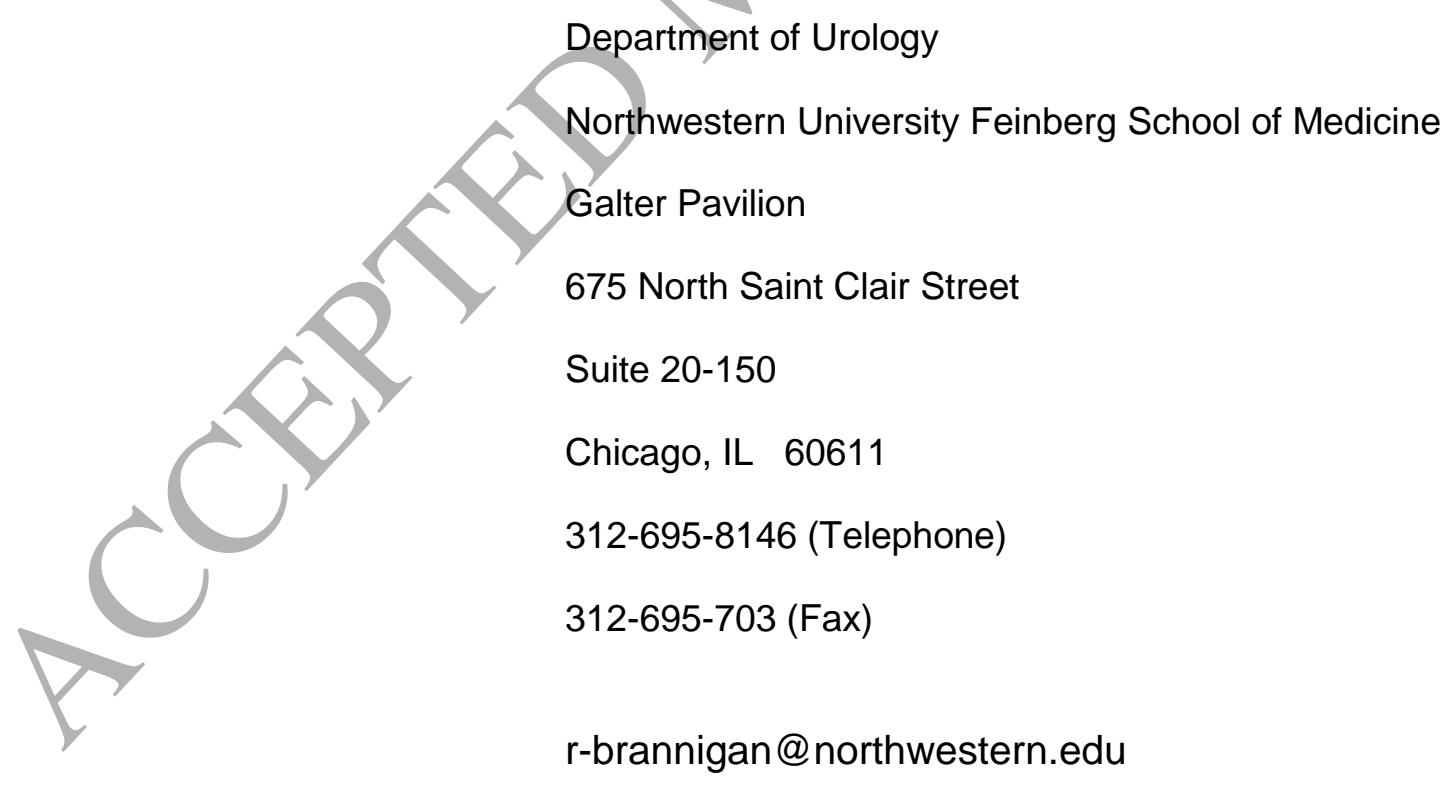

Conflicts of Interest: None for any author

This is the author's manuscript of the article published in final edited form as:

Lewis, K. C., Lam, I., Nieb, J., Lam, G., Desai, A. S., Mazur, D. J., ... Brannigan, R. E. (2018). Inconsistent Adoption of World Health Organization V (2010) Semen Analysis Reference Ranges in the United States Six Years After Publication. Urology. https://doi.org/10.1016/j.urology.2018.09.041 


\section{Abstract}

Objective:

To determine the percentage of laboratories in the United States that have adopted the World Health Organization 2010 (WHO 5) semen analysis (SA) reference values six years after their publication.

Methods:

Laboratories were identified via three approaches: using the Clinical Laboratory Improvement Amendments (CLIA) website, the CDC's 2015 Assisted Reproductive Technology Fertility Clinical Success Rate Report, and automated web searches. Laboratories were contacted by phone or email to obtain de-identified SA reports and reference ranges.

Results:

We contacted 617 laboratories in 46 states, of which $208(26.7 \%)$ laboratories in 45 states were included in our analysis. 132 (63.5\%) laboratories used WHO 5 criteria, 57 (27.4\%) used WHO 4 criteria, and 19 (9.1\%) used other criteria. WHO 5 criteria adoption rates varied by geographic region, ranging from $87.5 \%(35 / 40)$ in the Midwest to $50.0 \%(33 / 66)$ in the West. There was a greater adoption rate of WHO 5 reference values in academic affiliated (23/26, $88.5 \%)$ compared to non-academic affiliated laboratories $(110 / 182,60.4 \%)(P=0.028)$.

Conclusion: 
While the majority of laboratories have adopted WHO 5 criteria following its release six years ago, a large percentage (36.5\%) use what is now considered outdated criteria. This variability could result in the characterization of a male's semen values as being "within reference range" at one center and "outside of reference range" at another. This inconsistency in classification may result in confusion for the both patient and physician and potentially shift the burden of infertility evaluation and treatment to the female partner.

\section{INTRODUCTION}

Approximately $15 \%$ of couples experience difficulty conceiving; male factor infertility problems have been found to be solely responsible in $20 \%$, and contributory along with female factors in another $30-40 \%$ of cases[1]. Semen analysis (SA) is typically the first step in evaluating the male partner in couples seeking infertility treatment. Abnormalities in the SA prompt further workup for male factor infertility problems, while a normal SA often results in pursuing possible female factor infertility problems[2-6].

There is currently no set of reference ranges for SA which has been universally adopted. Among other causes, this lack of standardization leads to variable inter- and intra-laboratory reliability [7-10]. Many laboratories use the World Health Organization (WHO) reference ranges, which provide reference value recommendations for semen volume, sperm concentration, sperm motility, and \% normal sperm morphology. The adoption rates of the previous 1999 edition (WHO 4) criteria were found to be as low as $23 \%$ according to a study published 10 years after its release[11]. 
The WHO 5 reference values were developed by Cooper et al. by analyzing SA results from a large sample of men with proven fertility $[12,13]$. The data was acquired from multiple centers across diverse populations to identify reference intervals for values of semen parameters which can be extrapolated to the general population of fertile men. The reference values reflect the lower fifth centile (with $95 \%$ confidence intervals) of the study cohort. Since its publication in 2010, many publications have critiqued the changes applied to the WHO 4 criteria[14-18].

It is unknown how many laboratories utilize the WHO 5 reference values, now eight years after their release. We hypothesize that a substantial percentage of laboratories performing SA in the United States have not adopted WHO 5 criteria. This study attempts to determine the percentage of laboratories that have adopted the WHO 5 criteria based on a sampling of laboratories performing SA in the United States.

\section{MATERIALS AND METHODS}

A three-prong methodology was developed to identify laboratories. First, Clinical Laboratory Improvement Amendments (CLIA) laboratories were identified for query through the CLIA website (https://wwwn.cdc.gov/clia/Resources/LabSearch.aspx) using a search of the laboratory name with the terms: "andrology," "infertility," "semen analysis," or "reproductive" to identify laboratories that perform SA in the United States. Information extracted included the laboratory name, accreditation, street address, email address, and phone number. Second, each of the labs listed in the CDC's 2015 Assisted Reproductive Technology Fertility Clinical Success Rates Report were considered and duplicates identified through method one were excluded. Finally, automated web searches were conducted to gather a list of one hundred candidate websites per search, using query phrases including "semen analysis," "seminogram," 
"andrology," "infertility," "sperm count," "sperm motility," and "sperm morphology." The complete text was retrieved from each website and parsed for entity extraction using a natural language API. From the extracted text data, potential names of SA laboratories were identified from the list of phrases linked to the "organization" entity type using an algorithm analyzing whether the surrounding text addressed the topic of SA. Laboratories were cross-referenced with those gathered from methods one and two, and duplicates were excluded. Laboratories were contacted first by email, and then by phone if they did not respond or did not have an email listed. De-identified SA reports or reference ranges from responding laboratories were then obtained. Laboratories were excluded from the study if they did not perform SA, did not respond to our inquiry, the phone number was incorrect, or if the phone number was a duplicate. Each laboratory was counted only once in the analysis, regardless of the number of fertility centers it served. Laboratory affiliation with an academic center was determined based on an internet search with the laboratory's name and address.

The rate of WHO criteria adoption was computed by comparing reference range values and SA reports to WHO criteria (Table 1). Laboratories were also stratified by geographic region (West, Midwest, South, and Northeast) of the United States. Statistical analysis was performed using SPSS software (Version 21, IBM Corporation, Armonk, N.Y.). A chi-square test was used to compare the rate of WHO 5 criteria adoption between academic affiliated and non-academic affiliated laboratories. Significance was set at a $p$ value of $<0.05$.

\section{$\underline{\text { RESULTS }}$}

Our search resulted in 697 laboratories in 46 states. Four hundred eighty nine $(70.2 \%)$ laboratories were excluded from the analysis: 319 (65.2\%) did not reply to our inquiry, $36(7.4 \%)$ were wrong numbers, 80 (16.4\%) were duplicate laboratories or fertility centers using a 
laboratory that had already been contacted in our study, and 54 (7.7\%) did not perform SA. We obtained SA reference ranges from 208 (29.8\%) laboratories in 45 states. Our search resulted in $64(9.2 \%)$ academic affiliated laboratories and 633 (90.8\%) non-academic affiliated laboratories, of which we obtained reference values from 26 (40.6\%) and $182(28.8 \%)$ laboratories, respectively.

Our results included reference values from 66 (31.7\%) laboratories located in the West (MT, NM, AZ, CA, CO, HI, ID, NV, OR, UT, WA), 40 (19.2\%) in the Midwest (NE, SD, WI, IL, IN, KS, MI, MN, MO, OH), 65 (31.7\%) in the South (OK, LA, AL, AR, DC, DE, FL, GA, KY, MD, MS, SC, TN, TX, VA), and 37 (17.8\%) in the Northeast (NC, WV, CT, MA, NH, NJ, NY, PA, RI).

WHO 5 criteria were utilized by 132 (63.5\%) laboratories, while 57 (27.4\%) used WHO 4 criteria. 19 (9.1\%) laboratories did not comply with either WHO 4 or WHO 5 criteria; their reference values included WHO 3 criteria $(n=6)$ or modified WHO criteria $(n=11)$ (Table 2). 2 labs (1\%) stated they used parameters other than the WHO 4 or WHO 5, but would not specify the reference values used (Table 2). WHO 5 adoption rates varied greatly by geographic region, with $87.5 \%$ (35/40) in the Midwest, 61.5\% (24/39) in the Northeast, 60.3\% (38/63) in the South, and $50.0 \%(33 / 66)$ in the West (Figure 1). WHO 5 criteria adoption differed among academic centers $(23 / 26,88.5 \%)$ compared to non-academic centers $(110 / 182,60.4 \%)(p=0.028)$.

\section{DISCUSSION}

Semen analysis remains an essential step in diagnosing a male factor infertility problem. The SA results help guide the workup and treatment of a couple experiencing infertility. The AUA Best Practice Statement[6] entitled, "Optimal Evaluation of the Infertile Male," recommends a full evaluation of the male in the setting of two abnormal screening SA, while a normal SA will often result in the burden of the workup falling to the female. Laboratories performing SA are 
held to federal CLIA regulations, but are not required to conform to any specific criteria for their reference values[19]. The WHO 5 reference values are recommendations based on empiric evidence from data pooled from multiple studies across different populations. The WHO 5 recommendations recognize that there may be variations in normal semen analysis parameters by region, as was found by Jorgensen et al.,[20] and recommend that laboratories should consider preparing their own reference ranges to account for the regional variation. However, there is currently not enough data to accurately define individual reference ranges by region[12].

In the absence of specific regional data, most laboratories utilize the reference ranges published by the WHO, but many do not adopt the most up to date recommendations. In 2010, Penn et al.[11] found that only $23 \%$ of laboratories that they surveyed in the United States had adopted the recommended WHO 4 criteria 10 years after its publication. There are many different components of inter- and intra-laboratory standardization for SA, and the reference values are only one part of the process. Other problems that have been noted in the literature include lack of standardization in specimen preparation, variation in the technique used to evaluate the specimen, and lack of internal and external quality control measures[7-10, 21]. In 2005, Riddell et al.[22] reported that only $5 \%$ of the laboratories surveyed in the United Kingdom used the techniques set out in the WHO 4 recommendations for assessing sperm morphology. The inconsistency across laboratories in the various domains highlights the problem addressed in this study: a lack of standardization amongst laboratories leads to variable categorizations of men as within the reference ranges in one laboratory and outside the reference ranges in another.

The main criticism of the WHO 5 reference values is that they were developed from the SA of fertile men only. The implications of this are twofold: approximately $5 \%$ of fertile men will be outside the reference ranges, and a man with SA values within the reference ranges, but no pregnancy for over one year, is still subfertile. The SA measures concentration, motility, and 
appearance of sperm, which are used as surrogate screening tests for fertility, but does not measure fertility directly. Despite criticisms however, the WHO 5 criteria are lauded for providing the first evidence-based reference ranges for SA parameters and for providing techniques to help standardize laboratory results[23, 24].

The WHO 5 reference values decreased the lower limit of the reference ranges, resulting in classification of some men as within the reference ranges who would otherwise have been outside the reference ranges under the WHO 4 criteria[23, 25, 26]. Murray et al.[15] found that 103 of $501(15 \%)$ patients on retrospective analysis would have had one or more parameter outside the reference range according to WHO 4 criteria while having all the parameters within the reference ranges with WHO 5 criteria. It may be argued that the lower reference values in WHO 5 reflect a gradual decline in the fecundity which has been described over the past 3 decades[27], however the overall decline in semen parameters has been widely disputed, and high quality data to confirm this hypothesis is lacking[28, 29].

In collecting data from 208 laboratories, this study is the largest of its kind. These data confirm our hypothesis: just over one-third (36.5\%) of the surveyed laboratories did not adopt the WHO 5 reference ranges. Interestingly, this is a substantially lower percentage than previous reports of WHO 4 criteria adoption in 2010[11]. This difference could, in part, be explained by the fact that these reference ranges are "evidence-based" and thus might be considered by some to be more clinically relevant than previous criteria. It is also possible that there was not a large change from the 1992 (WHO 3) criteria[30] to the WHO 4 criteria. Therefore, laboratories may have felt less inclined to adopt WHO 4 reference ranges than when presented with a larger change in the reference values as seen with WHO 5 criteria[11]. While it is conceivable that greater standardization in the reference values might be a step towards higher reliability amongst laboratories, the fact that the WHO 5 reference ranges were 
generated without the consideration of any data from infertile male patients remains a substantial limitation and point of contention among many experts.

We also found a discrepancy in rates of WHO 5 criteria adoption in geographic regions across the country, ranging from $87.5 \%$ adoption in the Midwest to only $50 \%$ adoption in the West. Awareness of geographical differences is important for clinical practice as these results suggest that patients are more likely to have their SA performed at a laboratory using the WHO 5 reference values if they are in the Midwest or the Northeast than if they are in the West or the South. Additionally, a larger percentage of academic affiliated laboratories were found to have adopted the WHO 5 criteria, which reached statistical significance when compared to nonacademic affiliated laboratories. However, the disproportionate number of academic and nonacademic affiliated laboratories must be considered when interpreting this finding.

The findings of this study are important to consider as a lack of standardized reference values can result in a patient being considered within the reference ranges in one laboratory, and outside the reference ranges in another laboratory, even in the same geographic region. This inconsistency can lead to anxiety and confusion for both the patient and clinician. The confusion can impact the management of the patient and his partner depending when or where they seek treatment and is especially crucial with a time-sensitive issue, such as infertility. This is vital for providers to take into account when interpreting SA results and counseling their patients on the next steps of the couple's infertility workup.

The limitations of this study need to be considered when interpreting the results. Laboratories were given the option to provide a sample SA, or to provide the reference values that they use. In a few cases, laboratories reported that they use WHO 5 criteria, while the reference values they provided were actually in concordance with the WHO 4 criteria. These laboratories were categorized appropriately in our study as utilizing WHO 4 criteria, but it is 
important for physicians to note that when queried on their reference ranges, there may be a discrepancy between the reported criteria being followed and the actual reference values being used.

The search of the CLIA website was conducted based on a search of laboratory names, which resulted in laboratories without these terms in their name being excluded from our study. Though an attempt to mitigate unnecessary exclusion by utilizing the 2015 Assisted Reproductive Technology Fertility Clinical Success Rates Report and by developing a natural language API, a risk of exclusion persists. This, as well as our sample size of $n=208$ could introduce bias into our results. The lower percentage of academic affiliated laboratories surveyed could result in under- or over-representing the WHO 5 adoption rates among academic compared to non-academic affiliated laboratories. A larger sample size could improve the generalizability of the data. However, the laboratories were selected randomly across 45 states, with a close distribution among the geographical regions, which decreases the likelihood of sampling bias.

An additional limitation is that this study only examined reference ranges, and did not address any of the other areas that can result in inter- and intra- laboratory variability, such as sample preparation, examination technique, and quality control measures. Morphology, in particular has been found to be a large contributor to intra-laboratory variability. Both the WHO 4 and WHO 5 reference ranges are based on the recommended strict morphology criteria[31], however it is unclear how many laboratories are using this criteria, even if reporting the WHO 4 or 5 reference ranges $[8,9]$. These issues are addressed in the WHO 5 recommendations, and measuring compliance with these metrics is an important area of future study.

With so many laboratories performing SA, it may prove difficult to achieve standardization across laboratories. However, given how crucial the SA is for the infertility 
workup of the couple, achieving SA reliability among laboratories should be a priority. Also, given the variability in SA among fertile men, large regional studies are needed to more accurately characterize reference ranges and appropriate treatment thresholds in order to avoid undue placement of the burden for diagnosis and care on the female partner.

\section{CONCLUSION}

While the majority of laboratories in the United States have adopted WHO 5 reference range criteria, a large percentage (36.5\%) still use other criteria six years after the WHO 5 criteria were published. This variability could result in a male patient being characterized as "fertile" in one center and "subfertile" in another, leading to inconsistencies in treatment, and potentially shifting the burden of the infertility workup and interventions to the female. This discrepancy is particularly relevant given that, contrary to earlier standards, the WHO 5 criteria were developed from a population of normal, fertile males and did not contain infertile males. The current lack of consensus amongst laboratories in SA reference ranges could thus substantially impact the management of the male patient and his partner, simply depending on where they seek treatment. While large regional studies are needed to develop region-specific reference ranges, more consistent adoption of existing reference values would help to standardize the categorization of males and the subsequent approach to couples experiencing infertility. 


\section{REFERENCES}

1. Thonneau, $\mathrm{P}$., et al., Incidence and main causes of infertility in a resident population $(1,850,000)$ of three French regions (1988-1989). Hum Reprod, 1991. 6(6): p. 811-6.

2. Sharlip, I.D., et al., Best practice policies for male infertility. Fertil Steril, 2002. 77(5): p. $873-82$.

3. Sigman, M. and J.P. Jarow, Endocrine evaluation of infertile men. Urology, 1997. 50(5): p. 65964.

4. Pavlovich, C.P., et al., Evidence of a treatable endocrinopathy in infertile men. JUrol, 2001. 165(3): p. 837-41.

5. $\quad$ de Kretser, D.M., Male infertility. Lancet, 1997. 349(9054): p. 787-90.

6. Jarow, J.P., et al., The Optimal Evaluation of the Infertile Male: AUA Best Practice. American Urological Association Education and Research, Inc., 2010. p. 7-8.

7. Chong, A.P., C.A. Walters, and S.A. Weinrieb, The neglected laboratory test. The semen analysis. J Androl, 1983. 4(4): p. 280-2.

8. Keel, B.A., How reliable are results from the semen analysis? Fertil Steril, 2004. 82(1): p. 41-4.

9. Keel, B.A., et al., Lack of standardization in performance of the semen analysis among laboratories in the United States. Fertil Steril, 2002. 78(3): p. 603-8.

10. Ombelet, W., et al., Results of a questionnaire on sperm morphology assessment. Hum Reprod, 1997. 12(5): p. 1015-20.

11. Penn, H.A., et al., National semen analysis reference range reporting: adherence to the 1999 World Health Organization guidelines 10 years later. Fertil Steril, 2011. 95(7): p. 2320-3.

12. World Health Organization. WHO Laboratory Manual for the Examination and Processing of Human Semen, 5th ed. Geneva. WHO Press; 2010. p. 223-225.

13. Cooper, T.G., et al., World Health Organization reference values for human semen characteristics. Hum Reprod Update, 2010. 16(3): p. 231-45.

14. World Health Organization. Laboratory manual for the examination of human semen and spermcervical mucus interaction. 4th ed. New York: Cambridge University press, 1999. p. 60-62.

15. Murray, K.S., et al., The effect of the new 2010 World Health Organization criteria for semen analyses on male infertility. Fertil Steril, 2012. 98(6): p. 1428-31.

16. Esteves, S.C., et al., Critical appraisal of World Health Organization's new reference values for human semen characteristics and effect on diagnosis and treatment of subfertile men. Urology, 2012. 79(1): p. 16-22.

17. Skakkebaek, N.E., Normal reference ranges for semen quality and their relations to fecundity. Asian J Androl, 2010. 12(1): p. 95-8.

18. van der Steeg, J.W., et al., Role of semen analysis in subfertile couples. Fertil Steril, 2011. 95(3): p. 1013-9.

19. College of American Pathologists. Standards for reproductive laboratory accredidation. 2013 edition. p. 40-48.

20. Jorgensen, N., et al., Regional differences in semen quality in Europe. Hum Reprod, 2001. 16(5): p. 1012-9. 
21. Lu, J.C., et al., A survey on the status of semen analysis in 118 laboratories in China. Asian J Androl, 2010. 12(1): p. 104-10.

22. Riddell, D., A. Pacey, and K. Whittington, Lack of compliance by UK andrology laboratories with World Health Organization recommendations for sperm morphology assessment. Hum Reprod, 2005. 20(12): p. 3441-5.

23. De Jonge, C., Semen analysis: looking for an upgrade in class. Fertil Steril, 2012. 97(2): p. 260-6.

24. Haidl, G., New WHO-reference limits-revolution or storm in a teapot? Asian J Androl, 2011. 13(2): p. 208-11.

25. Centola, G.M., Determination of male infertility: is the 2010 WHO reference range helpful or confusing? Fertil Steril, 2012. 98(6): p. 1416-7.

26. Menkveld, R., Clinical significance of the low normal sperm morphology value as proposed in the fifth edition of the WHO Laboratory Manual for the Examination and Processing of Human Semen. Asian J Androl, 2010. 12(1): p. 47-58.

27. Carlsen, E., et al., Evidence for decreasing quality of semen during past 50 years. Bmj, 1992. 305(6854): p. 609-13.

28. Handelsman, D.J., Estrogens and falling sperm counts. Reprod Fertil Dev, 2001. 13(4): p. 317-24.

29. Andolz, P., M.A. Bielsa, and J. Vila, Evolution of semen quality in North-eastern Spain: a study in 22,759 infertile men over a 36 year period. Hum Reprod, 1999. 14(3): p. 731-5.

30. World Health Organization. WHO Laboratory Manual for the Examination of

Human Semen and Sperm-cervical Mucus Interaction, 3rd edn. Cambridge:

Cambridge University Press, 1992. p. 107.

31. Menkveld, R., et al., The evaluation of morphological characteristics of human spermatozoa according to stricter criteria. Hum Reprod, 1990. 5(5): p. 586-92. 


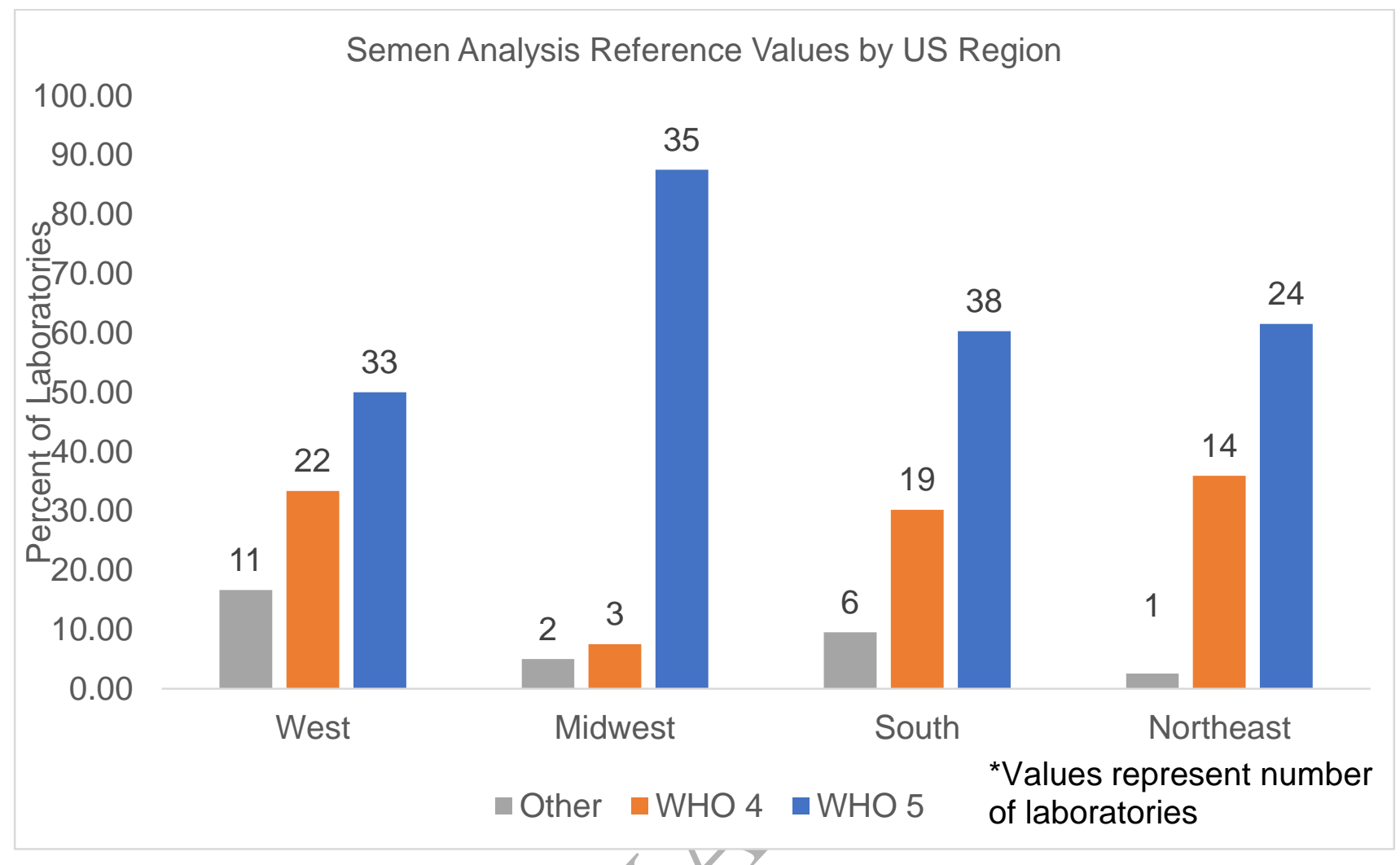

FIGURE 1: Semen Analysis Rarameter Reference Values by US Region.

Adoption rates of $\mathrm{WHO}_{5}, \mathrm{WHO} 4$, or other reference ranges stratified by region of the United States. 
TABLE 1: WHO Semen Analysis Reference Ranges

\begin{tabular}{|l|c|c|c|}
\hline Semen Parameter & WHO 3 (1992) & WHO 4 (1999) & WHO 5 (2010) \\
\hline Volume $(\mathrm{mL})$ & 2.0 & 2.0 & $1.5(1.4-1.7)$ \\
\hline Total sperm number $\left(10^{6}\right)$ & 40 & 40 & $39(33-46)$ \\
\hline Semen Concentration $\left(10^{6} / \mathrm{mL}\right)$ & 20 & 20 & $15(12-16)$ \\
\hline Progressive motility (\% progressive) & 50 & 50 & $32(31-34)$ \\
\hline Normal morphology $(\%)$ & 30 & 15 & $4(3.0-4.0)$ \\
\hline
\end{tabular}

TABLE 2: Semen Analysis Reference Ranges Utilized by Laboratory

\begin{tabular}{|l|c|}
\hline Semen Analysis Reference Ranges Utilized by Laboratory & $\begin{array}{l}\text { Number (\%) of } \\
\text { Laboratories }\end{array}$ \\
\hline WHO 5 criteria & $132(63.5 \%)$ \\
\hline WHO 4 criteria & $57(27.4 \%)$ \\
\hline Other & $19(9.1 \%)$ \\
\hline $\begin{array}{l}\text { WHO 3 criteria } \\
\text { WHO 4 volume and concentration, WHO 5 } \\
\text { morphology, progressive motility 40\% }\end{array}$ & $4(1.9 \%)$ \\
\hline $\begin{array}{l}\text { WHO 5 volume and concentration, WHO 4 } \\
\text { morphology, progressive motility 40\% }\end{array}$ & $5(2.4 \%)$ \\
\hline $\begin{array}{l}\text { WHO 4 volume and WHO 5 morphology } \\
\text { WHO 5 concentration and morphology, WHO 4 } \\
\text { volume, progressive motility 40\% }\end{array}$ & $1(0.5 \%)$ \\
\hline \begin{tabular}{l} 
Other criteria, unspecified \\
\hline
\end{tabular} & $2(0.5 \%)$ \\
\hline
\end{tabular}


Editorial Comment on "Inconsistent Adoption of World Health Organization V (2010) Semen Analysis Reference Ranges in the United States Six Years After Publication"

\section{R. Matthew Coward, MD FACS}

Department of Urology, University of North Carolina School of Medicine, Chapel Hill, NC UNC Fertility, Raleigh, NC

Corresponding Author:

R. Matthew Coward, MD FACS

2113 Physician's Office Building, CB\#7235

170 Manning Drive

Chapel Hill, NC 27599-7235

Phone: 919-966-8217

Fax: 919-966-0098

mcoward@med.unc.edu

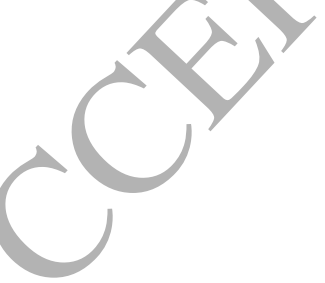

The inconsistent adoption of the WHO $5^{\text {th }}$ edition (2010) semen analysis reporting standard by laboratories is a huge problem in clinical andrology. Although the study by [First author last name] and colleagues has a few methodological shortcomings, its simple design is particularly effective at conveying the magnitude of this widespread problem. For those in a reproductive medicine specialty practice it will not be surprising, but for the rest of the urology 
community it will be staggering to see that over a third of U.S. laboratories are performing semen analyses according to decades-old standards ${ }^{1}$.

While the $\mathrm{WHO} 5^{\text {th }}$ edition standard is not perfect, it is the best and most current standard adopted by laboratories and reproductive centers worldwide to characterize a semen analysis on the spectrum of normal fertility. To that point, it is important to understand that these "reference ranges" do not represent fertile (normal) versus infertile (abnormal) in the same way that a white blood cell count can either be normal or represent an abnormal leúkocytosis. Rather, the lower limit of normal for each parameter is in fact the $5^{\text {th }}$ percentile of men with normal fertility, therefore indicating where on the continuum of normal fertility a particular semen parameter may be. Unfortunately, while a complete blood count result from another laboratory can easily and reliably be interpreted by a clinician, we cannot always say the same about a semen analysis. There are multiple reasons for this, with one of the primary reasons being inconsistent laboratory reporting.

Prior to the most recently published reference range in the WHO $5^{\text {th }}$ edition (2010), the second most recent edition (1999) is now approaching 20 years old. It is not invalid to report a result alongside an outdated reference range; however, when labs do not follow the most current standard of reporting, it represents a "red flag" to the clinician that the lab may not only perform semen analyses infrequently, but also, more importantly, may be out of touch with modern andrology assessment techniques. The resulting drawback is particularly evident with sperm morphology, which went through a significant change when Kruger strict morphology was adopted for the $5^{\text {th }}$ edition. The light microscopy version of morphology assessment, which was part of the 1999 and earlier WHO reference standards, is now obsolete and relatively useless to clinicians. Nevertheless, this change in morphology reporting is likely a factor in why some laboratories have not adopted the most current standard. 
Not only are non-conforming labs out of touch and potentially less accurate, but results reported by such labs can lead to mischaracterizations of patients' reproductive potentials, missed diagnoses, and ultimately can heighten the barriers to male reproductive care. It is about time that CLIA-certified laboratories be required to conform to modern reference values in reporting semen analyses in order to maintain compliance.

References:

1. Place reference for present study (currently unknown): "Inconsistent Adoption of World Health Organization V (2010) Semen Analysis Reference Ranges in the United States Six Years After Publication" 
Editorial Reply URL-D-17-00506R1

Word count: 263

Robert E. Brannigan, MD

Professor of Urology

Northwestern Feinberg School of Medicine

676 N. St. Clair St.

Arkes 23-015

Chicago, IL 60611

r-brannigan@northwestern.edu

We appreciate the reviewer's comments regarding our manuscript. The inconsistent adoption of WHO $V(2010)$ semen analysis reference ranges can result in numerous unintended consequences, including inconsistencies in the diagnosis and treatment of male factor infertility depending simply on the location of the patient's evaluation. Patients commonly express confusion and dismay when being categorized as "normal" at one center and "infertile" at another, and this scenario can also result in uncertainty on the part of physicians unfamiliar with the field of reproductive medicine. We agree with the reviewer that more consistent adoption of contemporary WHO semen analysis reference ranges would greatly help rectify this situation and provide patients and clinicians alike with great clarity. However, we suspect that a primary reason for the inconsistent adoption of the WHO V reference ranges is the fact that these values do not represent a true boundary between the "fertile" and "infertile" states. Rather, these values are arbitrary cut-points selected at the $5^{\text {th }}$ centile value for each parameter, derived from data from an international cohort of fertile males. Given the fact that the contemporary refence values are not derived to differentiate fertile from infertile men, many centers continue to use the older reference ranges because they feel that they do a better job discriminating fertility from infertility. Until reference ranges that more clearly separate these two groups are implemented, inconsistent adoption will likely persist. Unfortunately, given the high degree of overlap in semen analysis values for fertile and infertile men, we suspect that such reference ranges will not be generated any time soon.

Robert E. Brannigan, MD 